\title{
MONITORAMENTO DA ADMINISTRAÇÃO DE MEDICAMENTOS INJETÁVEIS EM UNIDADE DE PRONTO ATENDIMENTO (UPA)
}

\section{Camila Mioko Ono Lopes}

Discente de Farmácia no Centro Universitário de Maringá (UNICESUMAR), Maringá (PR), Brasil.

E-mail: camila.onolopes@hotmail.com

\section{Talma Reis Leal Fernandes}

Doutora em Ciências Biológicas (Biologia Celular) pela Universidade Estadual de Maringá(UEM), Maringá (PR), Brasil; Docente no curso de Farmácia no Centro Universitário de Maringá.
RESUMO: A superlotação das Unidades de Pronto Atendimento (UPAs) tem se tornado uma preocupação frequente para gestores e profissionais da saúde, pois gera mais custo para o sistema de saúde e auxilia na sobrecarga das equipes de trabalho. $\mathrm{O}$ objetivo deste estudo foi caracterizar e analisar a administração de medicamentos injetáveis prescritos em Unidade de Pronto Atendimento da região noroeste do Paraná. Foi utilizado um estudo descritivo e exploratório no período de 01 de junho a 31 de agosto de 2013. A população em estudo constituiu-se de uma amostra aleatória de 285 pacientes. As prescrições foram avaliadas quanto à dose, via de administração e diluição, e o tempo médio de administração dos medicamentos foi monitorado. Para análise dos dados foi empregada estatística descritiva baseada na distribuição de frequências e medidas de tendência central. A via de administração foi informada em 99,3\% das prescrições; entretanto, em $95,8 \%$ dos casos a dose não foi informada. As soluções diluentes utilizadas foram compatíveis com os medicamentos prescritos, embora tenha sido observada uma grande variação nos volumes das diluições, havendo necessidade de intervenção farmacêutica. Observou-se que o tempo médio de administração de medicamentos não foi responsável pelo aumento no tempo de permanência do paciente no serviço de saúde.

PALAVRAS-CHAVE: Gestão em Saúde; Medicina de Emergência; Prescrição de Medicamentos.

\section{MONITORING THE ADMINISTRATION OF INJECTABLE MEDICINE IN FIRST AID CLINICS}

ABSTRACT. Overcrowding in first aid clinics (UPAs) is a great concern for health managers and professionals since it increases costs and overloads health teams. Current study characterizes and analyzes the administration of injectable medicines prescribed in first aid clinics in the northwestern region of the state of Paraná, Brazil. The descriptive and exploratory study was undertaken between 1st June and 31st August 2013 with a randomized selected population of 285 patients. Prescriptions were assessed according to dose, administration mode, dilution and the mean time for the administration of the medicine. Data were analyzed by descriptive statistics based on the distribution of frequencies and trend measures. Administration mode was informed in $99.3 \%$ of prescriptions, although the dose was not given in $95.8 \%$ of cases. Dilution solutions were compatible to the medicines even though there was a great variation in dilution volumes, which required pharmaceutical intervention. Average time in the administration of medicines did not cause an increase in the patient 's permanence time at the unit.

KEY WORDS: Health Management; Emergency Medicine; Prescription of Medicines. 


\section{INTRODUÇÃO}

Segundo a Portaria $\mathrm{n}^{\circ} 1.601$, de 07 de julho de 2011, as Unidades de Pronto Atendimento (UPAs) são classificadas como unidades de saúde de complexidade intermediária entre as Unidades Básicas de Saúde (UBS), o Programa de Saúde da Família (PSF) e a Rede Hospitalar. Funcionam 24 horas por dia nos sete dias da semana e têm como objetivo diminuir as filas dos prontos-socorros dos hospitais, destinando-se a receber pacientes em situações de emergência, com ou sem risco iminente de morte. Devem ainda acolher a demanda fazendo a triagem classificatória de risco, resolver os casos de média complexidade, estabilizar os casos graves e fazer a interface entre a atenção básica e as unidades hospitalares (SANTOS et al., 2003; BRASIL, 2011; MIRANDA et al., 2012; SANTOS, 2012).

Embora a "porta de entrada" do sistema público de saúde seja preferencialmente a Atenção Básica (postos de saúde, centros de saúde, unidades de Saúde da Família, etc.), para que ocorra o encaminhamento para outros serviços de maior complexidade, muitos usuários têm demonstrado persistente preferência pelos serviços clássicos de urgência, o que contribui para a superlotação dos mesmos, inclusive das UPAs. Assim, estes serviços acabam acolhendo pacientes de urgências, pacientes desgarrados da atenção primária e especializada e as urgências sociais (BRASIL, 2006a; BRASIL, 2006b; O'DWYER, 2010; ACOSTA, 2012).

A superlotação dos prontos atendimentos, além de provocar um óbvio desgaste, devido à sobrecarga de trabalho, causa ainda um sentimento de desperdício da vocação maior do serviço que seria salvar vidas, bem como de subutilização do alto preparo técnico dos profissionais (POLL; LUNARDI; LUNARDI FILHO, 2008).

Pode-se caracterizar a superlotação de forma geral por ocupação de todos os leitos de emergência; presença de pacientes acamados nos corredores; tempo de espera para atendimento acima de uma hora; tensão das equipes de trabalho desses serviços; grande pressão para novos atendimentos. E o principal marcador da superlotação é o aumento do tempo de permanência do paciente nos serviços de primeiro atendimento e a falta de leitos a principal causa, o que leva ao atraso no diagnóstico e tratamento (BITTENCOURT; HORTALE, 2009).
O aumento da estadia no setor de saúde também pode ser ocasionado pelo uso inadequado de medicamentos que, além das consequências econômicas sérias às instituições, também trazem prejuízos à saúde dos pacientes. Estes erros envolvem desde a prescrição até a administração de medicamentos e são passíveis de prevenção (SILVA; CASSIANI, 2004; SILVA; SILVA; MIASSO, 2007; CRUCIOL-SOUZA; THOMSON; CATISTI, 2008).

O sistema de medicação tem início na prescrição médica e uma falha nessa etapa pode refletirse em problemas nas fases subsequentes, afetando a segurança do paciente, ou seja, a prescrição exerce papel fundamental no tratamento medicamentoso. A qualidade da informação encontrada nas prescrições, bem como a ausência de dados, pode levar a uma complexidade de problemas, com perda da qualidade da assistência farmacêutica. Os pacientes podem receber um medicamento em uma forma farmacêutica ou por uma via que não seja a mais adequada ao seu caso. As consequências podem variar desde o aumento no tempo de permanência no serviço até a elevação das taxas de mortalidade (SILVA; SILVA; MIASSO, 2007; CRUCIOLSOUZA; THOMSON; CATISTI, 2008).

Em relação aos erros ou inadequação da prescrição, Silva e Cassiani (2004) observaram problemas em relação à dosagem, via de administração, posologia, diluição e prescrição de medicamento inadequado. Miranda et al. (2012), além da diluição, observaram erros no tempo de infusão dos medicamentos. Esses últimos autores ainda demonstraram que intervenções farmacêuticas em UPA diminuíram significativamente erros de medicação, com consequente redução de custos, aumentando a qualidade do cuidado ao paciente e segurança em relação à terapia medicamentosa.

A intervenção farmacêutica citada por Miranda et al. (2012), em relação à análise da prescrição, pode ocorrer em sugerir troca de medicamento ao médico quando o mesmo prescreve medicamentos que não constam da relação dos padronizados pela entidade; identificar forma farmacêutica inexistente ou inadequada à administração; identificar prescrição de via de administração inadequada; identificar dose inexistente e/ou acima ou abaixo da dosagem usualmente prescrita; verificar frequência de administração inadequada ao medicamento; verificar modo de administração inadequado ao descrito em literatura; 
identificar diluente inadequado ou incompatível com o medicamento prescrito; verificar incompatibilidade entre medicamentos; identificar prescrição de medicamentos com a mesma ação farmacológica e medicamentos ilegíveis ou com descrição incompleta.

O relato da ocorrência de erros deve ser rápido para que sejam tomadas as devidas providências e, para que isso ocorra, as instituições devem ter uma ampla visão do sistema de medicação, possibilitando aos profissionais condições de análise e intervenções que garantam uma assistência responsável e segura ao paciente (SILVA; SILVA; MIASSO, 2007).

Segundo Cruciol-Souza, Thomson e Catisti (2008), a prescrição deve ser vista como um documento terapêutico de comunicação entre profissionais, sendo que o uso adequado deste instrumento pela equipe de saúde pode melhorar a utilização dos recursos financeiros da instituição. Daí a importância de se desenvolver protocolos e rotinas para a prescrição. $\mathrm{O}$ uso correto de medicamentos e a supervisão deste também podem reduzir o tempo de permanência do paciente na unidade de saúde.

Os dados e indicadores sugeridos pelo Ministério da Saúde para avaliar a qualidade dos serviços de saúde são de natureza operacional e de atendimento e deveriam ser mais amplamente computados na área da saúde. Indicadores que poderiam ser utilizados para avaliar a superlotação nas UPAs em relação ao uso inadequado de medicamentos poderiam ser, por exemplo, a percentagem de prescrições médicas preenchidas adequadamente (forma farmacêutica, dose, diluente e volume, etc.); tempo médio de espera dos pacientes antes e durante o atendimento (na triagem, na admissão, no consultório, no laboratório, nos exames, na medicação, etc.) (POLL; LUNARDI; LUNARDI FILHO, 2008; BITTENCOURT; HORTALE, 2009; MACHLINE; PASQUINI, 2011).

É comum a falta de padrões de mensuração da qualidade do atendimento prestado quando se busca responder às diretrizes do SUS. Assim, os indicadores gerenciais devem fornecer informações significativas para a condução dos processos e a tomada de decisão nos níveis estratégico, administrativo ou operacional (POLL; LUNARDI; LUNARDI FILHO, 2008; MACHLINE; PASQUINI, 2011).

Avaliar as prescrições e monitorar o tempo médio de administração de medicamentos como parte do tempo de permanência de um paciente no atendimento de saúde podem ser indicadores gerenciais úteis, contribuindo para obtenção de dados que possibilitem o desenvolvimento de protocolos e rotinas para a prescrição, o que poderá auxiliar na eliminação do desperdício de esforço humano, adaptação e treinamento dos atendentes às tarefas, como também no estabelecimento de normas de execução do trabalho.

Portanto, o objetivo deste trabalho foi caracterizar e analisar a administração de medicamentos injetáveis prescritos em uma Unidade de Pronto Atendimento (UPA) de uma cidade da região noroeste do Paraná.

\section{METODOLOGIA}

Foi realizado um estudo descritivo e exploratório no período de 01 de junho a 31 de agosto de 2013 em uma Unidade de Pronto Atendimento de uma cidade da região noroeste do Paraná. Essa unidade foi inaugurada em 25 de março de 2012 e se caracteriza pelo atendimento público de 24 horas nas especialidades de clínica médica e pediatria, com média de 10.000 atendimentos/mês (base de dados da instituição). O serviço conta com total de 20 leitos de observação e 04 leitos de emergência.

A população em estudo constituiu-se de uma amostra aleatória (probabilística com $\alpha=0,05$ e $\beta=0,10$ ) de 285 pacientes atendidos na UPA. Como critérios de inclusão foram selecionados pacientes adultos (acima de 18 anos), de ambos os gêneros, atendidos pela clínica médica e encaminhados à medicação com prescrição para medicamentos injetáveis. Foram excluídos os pacientes pediátricos e pacientes adultos com prescrição de medicamentos não injetáveis.

A coleta de dados foi realizada nos meses de junho, julho e agosto de 2013, com alternância dos dias da semana (segunda à sexta-feira) e turnos de atividades (manhã, tarde e noite), após liberação do Centro de Capacitação Permanente em Saúde (CECAPS) da Secretaria Municipal de Saúde e aprovação do Projeto pelo Comitê de Ética e Pesquisa do Unicesumar (CEP) (Parecer 296.472/13), tendo sido solicitado o consentimento informado dos participantes do estudo.

Para a coleta de dados utilizou-se como instrumento um formulário que contempla as informações 
de identificação dos usuários e as características das prescrições: idade, gênero, nome do medicamento ou medicamentos, dose, via de administração, diluição indicada, horário de início e fim da administração. Para a coleta do tempo de administração de medicamentos foi utilizado cronômetro digital da marca Technos ${ }^{\circledR}$.

O tempo de administração do medicamento foi considerado como o momento em que o paciente sentou-se na cadeira da sala de medicação (tempo inicial) até a finalização da administração do medicamento (tempo final), seja diretamente ou por infusão com diluente. Não foi incluído nesse tempo o preparo da medicação. Quando da administração direta de um ou mais medicamentos pelo mesmo acesso venoso, marcouse o tempo total da administração. Da mesma forma, quando da administração por duas vias, endovenosa e intramuscular, cronometrou-se o tempo total para a administração dos medicamentos.

A classificação farmacológica dos medicamentos foi realizada segundo o Anatomical Therapeutic Chemical (ATC) do WHO Collaborating Centre for Drug Statistics Methodology, órgão da Organização Mundial da Saúde (OMS) (WHO, 2013).

Para análise dos dados foi empregada estatística descritiva baseada na distribuição de frequências e medidas de tendência central. As diferenças entre médias ou proporções foram analisadas com teste Qui-Quadrado para as variáveis categóricas, teste t-Student para as variáveis dicotômicas e teste Kolmogorov-Smirnov para determinar a normalidade da distribuição nas variáveis contínuas. As associações entre essas variáveis e as medidas de desfechos foram analisadas por meio do teste Qui-Quadrado. Com a finalidade de verificar as relações entre o tempo de administração de medicamentos e o volume de diluição utilizou-se o teste estatístico ANOVA.

\section{RESULTADOS}

Do total de 285 prescrições de medicamentos injetáveis de pacientes avaliados, 60,7\% eram do sexo feminino, com mediana de 35 anos (18-92 anos) (p $<0,01)$. Apesar da diferenciação entre os gêneros na procura ao serviço, não foram observadas diferenças significantes entre eles em relação à administração de medicamentos.
Ao todo foram administradas 536 doses. O total de prescrições examinadas (285) relacionou 16 diferentes medicamentos cuja ordem de frequência encontra-se disposta na Tabela 1 . Os cinco medicamentos prescritos com maior frequência, totalizando $76,6 \%$ das indicações, foram dipirona; tenoxicam; metoclopramida; butilescopolamina + dipirona e ranitidina.

Tabela 1. Distribuição de frequência dos medicamentos injetáveis administrados na UPA (jun-ago, 2013)

\begin{tabular}{l|c|c}
\hline Medicamento & Frequência* & $\%$ \\
\hline Dipirona & 127 & 23,7 \\
Tenoxicam & 109 & 20,3 \\
Metoclopramida & 74 & 13,8 \\
Butilescopolamina + Dipirona & 59 & 11,0 \\
Ranitidina & 42 & 7,8 \\
Diclofenaco & 32 & 6,0 \\
Tramadol & 29 & 5,4 \\
Benzilpenicilina Benzatina & 22 & 4,1 \\
Dimenidrinato + Piridoxina + Glicose & 21 & 3,9 \\
+ Frutose & 13 & 2,4 \\
Hidrocortisona & 2 & 0,4 \\
Dexametasona & 2 & 0,4 \\
Aminofilina & 1 & 0,2 \\
Furosemida & 1 & 0,2 \\
Ceftriaxona & 1 & 0,2 \\
Fenobarbital & 1 & 0,2 \\
Diazepam & 536 & 100,0 \\
\hline Total & & \\
\hline
\end{tabular}

* p valor $<0,01$ (Teste qui-quadrado com $\mathrm{df}=15$ e $\alpha=0,05$ ).

Os medicamentos administrados foram classificados em subgrupos terapêuticos, segundo a classificação ATC (Anatomical Therapeutic Chemical) (WHO, 2013). Das 536 doses observou-se que 156 (29,15\%) foram de analgésicos; 141 (26,3\%) de antiinflamatórios e antirreumáticos; 133 (24,8\%) de digestivos; 42 (7,8\%) de antiácidos; 23 (4,3\%) de antibacterianos; 21 (3,9\%) de anti-histamínicos; 15 (2,8\%) de corticóides; 2 $(0,4 \%)$ de antiasmáticos; $1(0,2 \%)$ de diuréticos; 1 (0,2\%) de antiepiléticos; $1(0,2 \%)$ de antipsicóticos, com valor de $\mathrm{p}<0,01$ (Teste qui-quadrado com $\mathrm{df}=15$ e $\alpha=0,05$ ).

Das 285 prescrições, 177 apresentaram associações de medicamentos, totalizando 47 tipos de associações, destas apenas uma (2,13\%) apresentou interação medicamentosa indesejável. Considerando 
o impacto sobre o paciente, a interação foi de grau moderado (aminofilina + hidrocortisona) (INTERAÇÕES MEDICAMENTOSAS, 2013).

A administração dos medicamentos foi estudada nas suas características em termos de via utilizada, número de medicamentos administrados, entre outras particularidades que se encontram dispostas na Tabela 2.

Tabela 2. Aspectos da administração dos medicamentos injetáveis observados na UPA (jun-ago, 2013)

\begin{tabular}{|c|c|c|c|}
\hline Característica & Frequência & $\%$ & $p$ valor \\
\hline \multicolumn{4}{|l|}{ Via de administração } \\
\hline Endovenosa (EV) & 228 & 80,0 & \multirow{3}{*}{$<0,01^{*}$} \\
\hline Intramuscular (IM) & 44 & 15,4 & \\
\hline $\begin{array}{l}\text { Endovenosa e Intramuscular } \\
\text { (simultâneas) }\end{array}$ & 13 & 4,6 & \\
\hline \multicolumn{4}{|l|}{$\begin{array}{l}\text { Número de medicamentos na } \\
\text { administração }\end{array}$} \\
\hline Um & 107 & 37,5 & \multirow{4}{*}{$<0,01^{*}$} \\
\hline Dois & 130 & 45,6 & \\
\hline Três & 43 & 15,1 & \\
\hline Quatro & 5 & 1,8 & \\
\hline \multicolumn{4}{|l|}{$\begin{array}{l}\text { Informação de dose }{ }^{a} \text { na } \\
\text { prescrição }\end{array}$} \\
\hline Sim & 12 & 4,2 & \multirow{2}{*}{$<0,01 *$} \\
\hline Não & 273 & 95,8 & \\
\hline \multicolumn{4}{|l|}{$\begin{array}{l}\text { Informação da via de } \\
\text { administração na prescrição }\end{array}$} \\
\hline Sim & 283 & 99,3 & \multirow{2}{*}{$<0,01 * *$} \\
\hline Não & 2 & 0,7 & \\
\hline \multicolumn{4}{|l|}{ Tipos de administração EV } \\
\hline Bolus endovenoso ${ }^{\mathrm{b}}$ & 100 & 35,1 & \\
\hline Infusão intermitente ${ }^{c}$ & 121 & 42,4 & $<0,01 *$ \\
\hline Infusão contínua $^{\mathrm{d}}$ & 7 & 2,4 & \\
\hline $\begin{array}{l}\text { Volume médio injetado (média } \\
\pm \mathrm{dp} \text { ) em } \mathrm{mL}\end{array}$ & \multicolumn{2}{|c|}{$117,39( \pm 195,12)$} & $\begin{array}{c}< \\
0,01 * * *\end{array}$ \\
\hline Volume mediano injetado em $\mathrm{mL}$ & \multicolumn{2}{|l|}{20} & \\
\hline $\begin{array}{l}\text { Tempo médio de administração } \\
\text { (média } \pm \mathrm{dp} \text { ) em minutos }\end{array}$ & \multicolumn{2}{|c|}{$26,58( \pm 26,51)$} & $\begin{array}{c}< \\
0,01 * * *\end{array}$ \\
\hline $\begin{array}{l}\text { Tempo mediano de } \\
\text { administração em minutos }\end{array}$ & \multicolumn{2}{|l|}{10} & \\
\hline $\begin{array}{l}\text { Velocidade média de } \\
\text { administração (mL/min) }\end{array}$ & \multicolumn{2}{|l|}{4,41} & $\underset{0,01 * * * *}{<}$ \\
\hline \multicolumn{4}{|c|}{$\begin{array}{l}\text { *Teste qui-quadrado }(\alpha=0,05) ; * * \text { Teste t-Student }(\alpha=0,05) ; * * * \text { Tes- } \\
\text { te Kolmogorov-Smirnov }(\alpha=0,05) ; * * * * \text { Teste de correlação de Pear- } \\
\text { son }(\alpha=0,05) .{ }^{a} \text { Consideraram-se as doses prescritas com base na } \\
\text { concentração dos medicamentos. }{ }^{\mathrm{b}} \text { Bolus endovenoso=volumes até } \\
20 \mathrm{~mL} \text { administrados em até } 10 \text { minutos. }{ }^{c} \text { Infusão intermitente }=- \\
\text { volumes até } 500 \mathrm{~mL} \text { administrados em até } 1 \text { hora. }{ }^{\mathrm{d}} \text { Infusão contí- } \\
\text { nua }=\text { volumes até } 1000 \mathrm{~mL} \text { administrados em até } 12 \text { horas. }\end{array}$} \\
\hline
\end{tabular}

A maioria das prescrições, 283 (99,3\%), continha informação da via de administração, sendo que as vias injetáveis prescritas foram: endovenosa (EV); intramuscular (IM); e endovenosa + intramuscular (EV + IM). Houve um predomínio significativo da via EV com $80 \%$ de frequência, em relação aos $15,4 \%$ da via IM e $4,6 \%$ da associação das duas vias $(\mathrm{p}<0,01)$. Os tipos de administração EV foram: bolus endovenoso $(35,1 \%)$; infusão intermitente $(42,4 \%)$; e infusão contínua $(2,4 \%)$ (Tabela 2).

Os volumes e tempos considerados para a administração EV foram: bolus venoso - até $20 \mathrm{~mL}$ administrados em até 10 minutos; infusão intermitente - até $500 \mathrm{~mL}$ administrados em até 1 hora; e infusão contínua - até $1000 \mathrm{~mL}$ administrados em até 12 horas.

Nos dados referentes aos diluentes encontramos variação no volume da água para injeção com $3,0 \mathrm{ml}$; 4,0 mL; 8,0 mL; 10,0 mL; e $20 \mathrm{~mL}$. As soluções diluentes utilizadas para infusão foram: solução de cloreto de sódio 0,9\% (100 mL; $250 \mathrm{~mL} ; 500 \mathrm{~mL}$; e $1000 \mathrm{~mL}$ ) e solução de Ringer lactato.

O volume médio injetado foi de $117,39 \mathrm{~mL}$ ( \pm 195,12), com volume mediano de $20 \mathrm{~mL}(\mathrm{p}<0,01)$. O número de medicamentos variou de 1 a 4 por prescrição, sendo que houve predomínio da associação de 2 medicamentos em $130(45,6 \%)$ prescrições, seguido por 1 medicamento em 107 (37,5\%) (Tabela 2).

Os dados da Tabela 2 também permitem verificar que as informações nas prescrições não estavam completas, a dose que deveria ser administrada não foi indicada em 273 (95,8\%) prescrições, considerando-se dose como concentração do medicamento, ou seja, a quantidade capaz de provocar uma resposta terapêutica desejada no paciente.

Em relação ao tempo de administração de medicamentos, a média obtida foi de 26,58 minutos ( \pm $26,51)$, com tempo mediano de 10 minutos $(\mathrm{p}<0,01)$. A velocidade média de infusão foi de $4,41 \mathrm{~mL} /$ minuto $(\mathrm{p}<0,01)$ (Tabela 2).

O tempo médio gasto com a administração dos medicamentos, de acordo com a via de administração, é demonstrado na Tabela 3. Observa-se que o maior tempo médio, em minutos, foi obtido com a infusão contínua $\mathrm{EV}$; seguida da infusão intermitente EV; administração EV + IM; via IM e bolus EV. 
Tabela 3. Tempo médio gasto com a administração dos medicamentos, segundo a via de administração selecionada, observado na UPA (jun-ago, 2013)

\begin{tabular}{lcc}
\hline \multirow{2}{*}{ Via de administração } & \multicolumn{2}{c}{ Tempo (minutos) } \\
\cline { 2 - 3 } & média $\pm \mathrm{dp}$ & p valor* \\
\hline Intramuscular & $6 \pm 14$ & \\
Endovenosa & $30 \pm 27$ & \\
$\quad$ Bolo & $5 \pm 6$ & \\
$\quad$ Infusão intermitente & $49 \pm 17$ & \\
$\quad$ Infusão contínua & $75 \pm 25$ & \\
Endovenosa + Intramuscular & $30 \pm 20$ & \\
\hline
\end{tabular}

*Teste qui-quadrado com $\mathrm{df}=98$ e $\alpha=0,05$.

A Tabela 4 apresenta o tempo médio gasto com a administração dos medicamentos, segundo o número de medicamentos administrados, independente da via de administração.

Tabela 4. Tempo médio gasto com a administração dos medicamentos, segundo o número de medicamentos administrados, independente da via de administração, observado na UPA (jun-ago, 2013)

\begin{tabular}{ccc}
\hline & \multicolumn{2}{c}{ Tempo (minutos) } \\
\cline { 2 - 3 } Número de medicamentos & $\begin{array}{c}\text { média } \\
\pm \mathrm{dp}\end{array}$ & $\boldsymbol{p}$ v valor* \\
\hline 1 & $17 \pm 24$ & \\
2 & $27 \pm 26$ & \\
3 & $43 \pm 23$ & $<0,01$ \\
4 & $69 \pm 18$ & \\
\hline
\end{tabular}

*Teste qui-quadrado com $\mathrm{df}=147$ e $\alpha=0,05$.

Quando da associação de 4 medicamentos, o tempo de administração foi maior, na sequência encontramos o tempo da administração de 3 medicamentos, posteriormente associação de 2 medicamentos, e com menor tempo de administração, 1 medicamento.

A Figura 1 representa a associação entre $o$ tempo de administração dos medicamentos e o volume de diluição. Observa-se que existe uma correlação significativa entre o volume infundido e o tempo de administração do medicamento, ou seja, quanto maior o volume infundido, maior o tempo de administração do medicamento. Da mesma forma, volumes menores representam tempos de administração também menores.

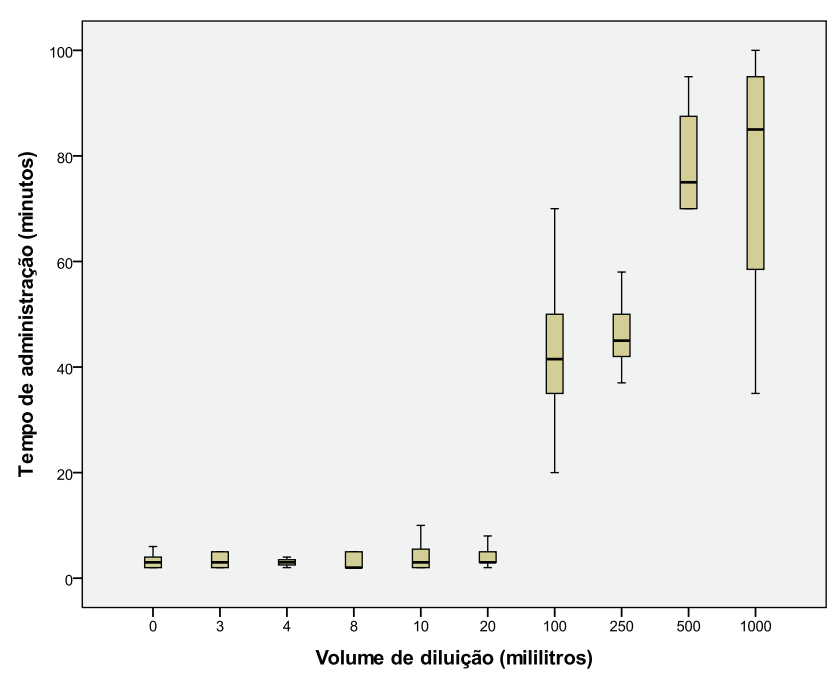

Figura 1. Associação entre tempo de administração dos medicamentos e o volume de diluição (diferença significativa entre as médias ao nível de $\mathrm{p}<0,01-$ ANOVA com $\mathrm{IC}=95 \%$ ).

\section{DISCUSSÃO}

A prevalência pela busca de atendimento ao serviço de saúde por mulheres pode ter explicação no estudo realizado por Gomes, Nascimento e Araújo em 2007. Esses pesquisadores analisaram as explicações presentes em discursos masculinos para a menor procura dos homens por serviços de saúde. Os resultados apontaram que o imaginário de ser homem pode aprisionar o masculino em amarras culturais, dificultando a adoção de práticas de autocuidado, podendo associá-lo a um ser fraco e inseguro.

A seleção de medicamentos injetáveis disponíveis pela unidade estudada mostra coerência com 0 preconizado pelo Ministério da Saúde. Dos 16 diferentes medicamentos prescritos na UPA, 11 constam da Lista de Medicamentos do Capítulo III do Anexo "Sistemas Estaduais de Urgência e Emergência - Regulamento Técnico" da Portaria GM n ${ }^{0} 2.048$, de 05 de novembro de 2002, que regulamenta os medicamentos que devem estar disponíveis nas unidades de emergência, como aminofilina; dexametasona; diazepam; diclofenaco; dipirona; fenobarbital; furosemida; hidrocortisona; metoclopramida; ranitidina; tramadol (BRASIL, 2006a).

Os medicamentos prescritos também estão compatíveis com os sintomas de doenças encontrados no estudo de Oliveira et al. (2011). Esses autores relataram que, independente da sazonalidade, de forma geral, as 
queixas mais comuns de usuários de unidades de pronto atendimento foram cefaléia, dor torácica, lombalgia e dor abdominal, o que explica o maior número de prescrições de analgésicos, anti-inflamatórios e antirreumáticos, além de digestivos.

Em relação às interações medicamentosas, apenas uma foi encontrada e de grau moderado. As interações moderadas são aquelas cujos efeitos podem causar uma piora no estado clínico do paciente, exigindo tratamento adicional, hospitalização ou aumento no período de internação (SEHN et al., 2003; INTERAÇÕES MEDICAMENTOSAS, 2013).

Considerando a via de administração, houve predomínio da infusão $\mathrm{EV}$ intermitente e em bolus em relação à infusão contínua. Uma injeção venosa por bolus caracteriza-se pela administração direta, ou através de um ponto de injeção no cateter, propiciando um nível de medicamento máximo quase imediato no sangue do paciente. $\mathrm{Na}$ infusão intermitente o paciente recebe medicamento endovenoso já diluído em períodos específicos de tempo, a intervalos variados, enquanto que a infusão contínua caracteriza-se pela administração de preparações medicamentosas já diluídas através de sistemas de perfusão com grandes volumes de infusão (PHILLIPS, 2001).

Segundo as bulas dos medicamentos e o Formulário Terapêutico Nacional (FTN) (BRASIL, 2010), todas as soluções diluentes utilizadas estavam compatíveis com os medicamentos prescritos. Entretanto, houve grande variabilidade no volume dos diluentes utilizados na UPA, constatando que as técnicas de diluição realizadas, embora não incorretas, podem não estar mantendo um padrão de operação.

Os volumes de diluentes não foram incorretos, entretanto, em alguns casos, poderiam ser utilizados volumes menores quando da administração EV intermitente ou mesmo utilizar-se da via por bolus EV ao invés da infusão (TRISSEL, 2008). Mas ao analisarmos o volume médio e mediano, deduzimos que essas alterações não interferiram de forma significativa no aumento do tempo de permanência do paciente na unidade de saúde.

No preparo de medicamentos devem ser aplicados vários princípios científicos que garantam $o$ resultado terapêutico esperado, para isto é necessário saber qual diluente usar, qual volume administrar de acordo com o acesso venoso e a dose correta. Faz-se essencial a adoção de práticas profissionais baseadas em protocolos de diluição, através de intervenção farmacêutica (CAMERINI; SILVA, 2011).

Silva et al. (2007) destacaram a importância da educação continuada dos profissionais envolvidos diretamente na execução do procedimento de administração de medicamentos, favorecendo uma melhor qualidade do cuidado prestado ao usuário. Nesses casos a presença do farmacêutico clínico é essencial, como demonstraram Miranda et al. (2012) no estudo realizado em Unidade de Pronto Atendimento, onde a intervenção farmacêutica gerou redução de custos e aumentou a qualidade do cuidado ao paciente $\mathrm{e}$ segurança em relação à terapia medicamentosa.

A falta de dados, como detectado nesta pesquisa em relação à dose, é considerada uma inadequação de prescrição de medicamentos o que pode acarretar em ocorrência de erros de medicação. Estudos comprovam que os tipos de erros mais frequentes de medicação estão na prescrição de medicamentos. Adotar medidas administrativas voltadas ao planejamento do sistema de medicação, como treinamentos e relatórios de ocorrência de erros de medicação, podem ser estratégias iniciais para minimizar esses problemas (SILVA; CASSIANI, 2004; SILVA; SILVA; MIASSO, 2007).

Esses erros poderiam ser reduzidos significativamente, por exemplo, se o farmacêutico revisasse as prescrições. Já foi observado através de pesquisa que intervenções farmacêuticas geram benefícios diretos ao paciente e também para a equipe interdisciplinar, uma vez que quase todas as recomendações realizadas pelo farmacêutico são aceitas por outros profissionais de saúde. A UPA é um setor dinâmico e as intervenções farmacêuticas realizadas pelo farmacêutico clínico podem trazer melhores resultados, garantindo segurança na terapia medicamentosa (MIRANDA et al., 2012).

Nestes dados algumas fragilidades do estudo não puderam ser superadas, pois, para a medição do tempo médio, não foram considerados os materiais utilizados para administração, bem como a experiência do profissional da enfermagem e dificuldades relativas aos pacientes.

O tempo médio de administração de 
medicamentos obtido na pesquisa foi de 26,58 minutos e este tempo foi maior para a infusão contínua EV e infusão intermitente, devido à utilização de maior quantidade de solução diluente. Da mesma forma, a associação de 4 ou 3 medicamentos também demandou um maior tempo de administração, o que era de se esperar, uma vez que um único medicamento exige apenas uma via, enquanto que a associação de dois ou mais medicamentos pode exigir vias diferentes de administração.

Ao avaliarmos que a somatória da administração de medicamentos por via IM e por bolus EV totalizou 144 $(50,5 \%)$ prescrições e que para este tipo de administração utilizaram-se volumes de até $20 \mathrm{~mL}$ e que, conforme mostra a Figura 1, o tempo de administração nestes casos é reduzido, não podemos considerar que a superlotação na UPA seja devido à permanência prolongada do paciente causada pela demora na administração de medicamentos. Outro dado que corrobora com esta afirmação é a mediana do tempo de administração de medicamentos de 10 minutos, evidenciada na Tabela 2 .

\section{CONCLUSÃO}

Os resultados desse trabalho detectaram erros de prescrição, fazendo-se necessária uma intervenção farmacêutica no sentido de revisar as mesmas. Sugerese o desenvolvimento de protocolos e rotinas para a prescrição na Unidade de Pronto Atendimento estudada, bem como cursos e treinamentos.

Através do monitoramento da administração de medicamentos injetáveis pode-se calcular o tempo médio de administração de medicamentos e os dados obtidos não evidenciaram que este seja o marcador responsável pelo aumento no tempo de permanência do paciente no serviço de saúde.

Para futuros estudos seria importante observar a gestão do trabalho interno da UPA em questão, como o serviço de classificação de risco e o tempo gasto em outras atividades: recepção, consultas, realização de exames, etc. Verificar também a saturação do limite operacional da unidade, identificando, por exemplo, falta de funcionários, exaustão das equipes de trabalho, estrutura física incapaz de atender a demanda, entre outros.

Outra possibilidade é que o paciente esteja procurando o serviço de pronto atendimento em situações não urgentes, embora esses serviços encontrem-se usualmente lotados, porque essas unidades reúnem um somatório de recursos como consultas, medicamentos, exames laboratoriais e radiológicos, procedimentos de enfermagem. Esses recursos satisfazem as necessidades do usuário. Provavelmente a atenção básica não deva estar sendo efetiva como porta de entrada para o sistema de saúde, evidenciando a fragilidade desse sistema.

Para que as Unidades de Pronto Atendimento sejam utilizadas apropriadamente há necessidade de revisão do sistema de saúde, melhorando a estrutura da atenção básica.

\section{REFERÊNCIAS}

ACOSTA, A. M. Usuários frequentes de um serviço de urgência: perfil e motivos de busca por atendimento. 2012. Tese (Mestrado em Enfermagem) - Universidade Federal do Rio Grande do Sul, Porto Alegre, 2012.

BITTENCOURT, R. J.; HORTALE, V. A. Intervenções para solucionar a superlotação nos serviços de emergência hospitalar: uma revisão sistemática. Cad. Saúde Pública, Rio de Janeiro, v. 25, n. 7, p. 1439-1454, 2009.

BRASIL. Ministério da Saúde. Entendendo o SUS. Brasília: Ministério da Saúde, 2006b.

BRASIL. Ministério da Saúde. Formulário terapêutico nacional: Rename 2010. 2. ed. Brasília: DF: Ministério da Saúde, 2010.

BRASIL. Ministério da Saúde. Política Nacional de Atenção às Urgências. 3. ed. Brasília: Ministério da Saúde, 2006a.

BRASIL. Ministério da Saúde. Portaria $n^{0} 1.601$, de 07 de julho de 2011. Estabelece diretrizes para a implantação do componente Unidades de Pronto Atendimento (UPA 24h) e o conjunto de serviços de urgência 24 horas da Rede de Atenção às Urgências, em conformidade com a Política Nacional de Atenção às Urgências. Diário Oficial da União, Brasília, DF, 8 jul. 2011.

CAMERINI, F. G.; SILVA, L. D. Segurança do paciente: análise do preparo de medicação intravenosa em 
Hospital da Rede Sentinela. Texto Contexto - Enferm., Florianópolis, v. 20, n. 1, p. 41-49, 2011.

CRUCIOL-SOUZA, J. M.; THOMSON, J. C.; CATISTI, D. G. Avaliação de prescrições medicamentosas de um hospital universitário brasileiro. Rev. Bras. Educ. Méd., Rio de Janeiro, v. 32, n. 2, p. 188-196, 2008.

GOMES, R.; NASCIMENTO, E. F.; ARAÚJO, F. C. Por que os homens buscam menos os serviços de saúde do que as mulheres? As explicações de homens com baixa escolaridade e homens com ensino superior. Cad. Saúde Pública, Rio de Janeiro, v. 23, n. 3, p. 565-574, 2007.

INTERAÇÕES MEDICAMENTOSAS c 2008-2012. Disponível em: < http://interacoesmedicamentosas.com. br>. Acesso em: 02 mar. 2013.

MACHLINE, C.; PASQUINI, A. C. Rede hospitalar nacional usa indicadores gerenciais na administração de suas unidades. O Mundo da Saúde, São Paulo, v. 35, n. 3, p. 290-299, 2011.

MIRANDA, T. M. M.; PETRICCIONE, S.; FERRACINI, F. T.; BORGES FILHO, W. M. Intervenções realizadas pelo farmacêutico clínico na unidade de primeiro atendimento. Einstein, São Paulo, v. 10, n. 1, p. 74-78, 2012.

O'DWYER, G. A gestão da atenção às urgências e o protagonismo federal. Ciênc. Saúde Coletiva, Rio de Janeiro, v. 15, n. 5, p. 2395-2404, 2010.

OLIVEIRA, G. N.; SILVA, M. F. N.; ARAÚJO, I. E. M.; CARVALHO-FILHO, M. A. Perfil da população atendida em uma unidade de emergência referenciada. Rev. LatinoAm. Enfermagem [online], v. 19, n. 3. Disponível em: $<$ http://www.scielo.br/pdf/rlae/v19n3/pt_14>. Acesso em: 02 mar. 2013.

PHILLIPS, L. D. Manual de terapia intravenosa. Tradução Pedreira, M. L. G. 2. ed. Porto Alegre: Artmed, 2001.

POLL, M. A.; LUNARDI, V. L.; LUNARDI FILHO, W. D. Atendimento em unidade de emergência: organização e implicações éticas. Acta Paul. Enferm. [online], São Paulo, v. 21, n. 3, p. 509-514, 2008.

SANTOS, J. S.; SCARPELINI, S.; BRASILEIRO, S. L. L.; FERRAZ, C. A.; DALLORA, M. E. L. V.; SÁ, M. F. S. Avaliação do modelo de organização da unidade de emergência do HCFMRP-USP, adotando, como referência, as Políticas Nacionais de Atenção às Urgências e de Humanização. Medicina, Ribeirão Preto, v. 36, p. 498-515, 2003.

SANTOS, N. C. Os novos formatos de gestão nos serviços de saúde e a efetivação de direitos: UPA/ Pajuçara, Natal/RN. 2012. Trabalho de Conclusão de Curso (Curso de Serviço Social) - Universidade Federal do Rio Grande do Norte, Natal, RN.

SEHN, R.; CAMARGO, A. L.; HEINECK, I.; FERREIRA, M. B. C. Interações medicamentosas potenciais em prescrições de pacientes hospitalizados. Infarma, Brasília, v. 15, n. 9-10, p. 77-81, 2003.

SILVA, A. E. B. C.; CASSIANI, S. H. B. Erros de medicação em Hospital Universitário: tipo, causas, sugestões e providências. Rev. Bras. Enferm., Brasília, v. 57, n. 6, p. 671-674, 2004

SILVA, B. K.; SILVA, J. S.; GOBBO, A. F. F.; MIASSO, A. I. Erros de medicação: condutas e propostas de prevenção na perspectiva da equipe de enfermagem. Rev. Eletr. Enf. [On line], v. 9, n. 3, p. 712-723, 2007. Disponível em: $<$ http://www.fen.ufg.br/revista/v9/n3/v9n3a11.htm > . Acesso em: 02 mar. 2013.

TRISSEL, L. A. Guia de bolso para fármacos injetáveis. 14. ed. Porto Alegre: Artmed, 2008.

WORLD HEALTH ORGANIZATION. Collaborating Centre for Drug Statistics Methodology. Anatomical Therapeutic Chemical (ATC) index with Definined Daily Doses (DDDs). Disponível em: <www.whocc.no/atcddd>. Acesso em: 31 out. 2013.

Recebido em: 29 de janeiro de 2015 Aceito em: 18 de março de 2015 